\title{
Direct Observation of Wet Biological Samples by Graphene Liquid Cell Transmission Electron Microscopy
}

\author{
Jungwon Park, ${ }^{\dagger, \ddagger}$ Hyesung Park, ${ }^{\S}$ Peter Ercius,,$"$ Adrian F. Pegoraro, ${ }^{\dagger, \ddagger}$ Chen Xu, ${ }^{\perp}$ Jin Woong Kim, ${ }^{\#, \nabla}$
} Sang Hoon Han, ${ }^{\bigcirc}$ and David A. Weitz ${ }^{*, \dagger, \neq}$

${ }^{\dagger}$ Department of Applied Physics, Harvard University, Cambridge, Massachusetts 02138, United States

${ }^{\ddagger}$ School of Engineering and Applied Sciences, Harvard University, Cambridge, Massachusetts 02138, United States

${ }^{\S}$ School of Energy and Chemical Engineering, Ulsan National Institute of Science and Technology, Ulsan 689-798, South Korea

"The Molecular Foundry, Lawrence Berkeley National Laboratory, Berkeley, California 94720, United States

${ }^{\perp}$ Rosenstiel Basic Medical Sciences Research Center, Brandeis University, Waltham, Massachusetts 02454, United States

\# Department of Applied Chemistry, Hanyang University, Ansan 426-791, South Korea

${ }^{\nabla}$ Department of Bionano Technology, Hanyang University, Ansan 426-791, South Korea

OAmore-Pacific Co. R\&D Center, Yongin, Gyeonggi-do 446-729, South Korea

\section{Supporting Information}

\begin{abstract}
Recent development of liquid phase transmission electron microscopy (TEM) enables the study of specimens in wet ambient conditions within a liquid cell; however, direct structural observation of biological samples in their native solution using TEM is challenging since low-mass biomaterials embedded in a thick liquid layer of the host cell demonstrate low contrast. Furthermore, the integrity of delicate wet samples is easily compromised during typical sample preparation and TEM imaging. To overcome these limitations, we introduce a graphene liquid cell (GLC) using multilayer graphene sheets to reliably encapsulate and preserve biological samples in a liquid for TEM observation. We achieve nanometer scale spatial resolution with high contrast using low-dose TEM at room temperature, and we use the GLC to directly observe the structure of influenza viruses in their native buffer solution at room temperature. The GLC is further extended to investigate whole cells in wet conditions using TEM. We also demonstrate the potential of the GLC for correlative studies by TEM and fluorescence light microscopy imaging.
\end{abstract}

KEYWORDS: Liquid phase TEM, graphene liquid cell, multilayer graphene, bioimaging, influenza viruses, epithelial cells

M ost microscopy studies in biological sciences aim to observe samples of interest directly in their native conditions, with the choice of technique determined by the benefits of each type of microscopy. ${ }^{1-3}$ Fluorescence optical microscopy provides excellent contrast by using fluorophores emitting photons at different wavelengths. ${ }^{4-6}$ It can be used to study the structure and resulting function of macromolecules in vitro and in vivo and can even achieve a maximum resolution of about $10 \mathrm{~nm}$ through the use of super resolution microscopy., Transmission electron microscopy (TEM) can inherently reach spatial resolution on the level of angstroms due to the nature of the imaging electron beam. ${ }^{9,10}$ However, traditional TEM is limited to studying specimens that are both fixed and dried, due to the incompatibility of preserving the structure of a realistic, wet sample in the high vacuum necessary for TEM. Cryo-TEM enables imaging under more realistic conditions by using wet samples that are flash-frozen either in a buffer solution or in their parental cellular matrix. ${ }^{11-15}$ Cryo-TEM has further benefited from the recent developments of electron lens aberration correctors and direct electron detectors, both of which enhance contrast and spatial resolution, which leads to a significantly reduced electron dose and reduced sample damage. ${ }^{16,17}$ However, sample preparation methods for cryoTEM have inherent drawbacks: structure alteration can still occur during fast-freezing; formation of crystalline ice particles within the amorphous ice film often compromises the integrity of frozen samples, and weak mechanical stability of the thin amorphous ice layer limits sample regions for efficient imaging. Most of the sample is wasted by the blotting process used to make a thin layer of water before freezing, and samples suspended in a buffer solution tend to be distorted by the surface tension at the solution-air interface.

An alternate approach is liquid phase TEM where the sample is embedded in a thin liquid layer confined between two electron beam transparent windows, such as silicon nitride $\left(\mathrm{Si}_{3} \mathrm{~N}_{4}\right)$. This has been applied to the study of whole eukaryotic cells, macromolecules, and viruses in their native wet condition. ${ }^{18-22}$ However, the imaging electron beam is

Received: April 26, 2015

Revised: June 10, 2015

Published: June 12, 2015 
scattered by the $\mathrm{Si}_{3} \mathrm{~N}_{4}$ window, which typically has a thickness of tens of nanometers, significantly reducing contrast in TEM images, and the windows can reduce imaging conditions due to charging. Accumulated charges on the $\mathrm{Si}_{3} \mathrm{~N}_{4}$ window due to its low electrical conductivity also contribute to reducing contrast in TEM images. Furthermore, the sample chamber volume is predefined during the fabrication process, and any excess liquid further increases scattering, which limits the resolution of lowmass samples in a typical liquid cell TEM and is limited by the maximum extent of the object of interest. Therefore, for direct study of wet biological samples with TEM, we need a reliable method to prepare liquid cells using two windows that are lowscattering, thin, and that encapsulate biological samples in a minimum amount of liquid. Ideally, a single layer graphene could be used as the window material for liquid cells to minimize unwanted scattering while also reducing charge accumulation from the imaging electron beam. Graphene windows have recently been utilized to observe in situ dynamics of nanocrystals and to perform spectroscopic elemental analysis in liquid-phase TEM. ${ }^{23-26}$ However, direct structural observation of biological samples composed primarily of low-mass materials in liquid TEM remains challenging and faces several hurdles. The dimension of the liquid sample that can be captured between two graphene sheets is not well controlled since graphene is composed of multiple domains of the scale of microns, and defects between these domains can contribute to leaking of a liquid sample in the high vacuum of a TEM. In addition, single atom thickness makes liquid cell fabrication extremely delicate, and preparing reliable liquid cells that protect the sample integrity during the prolonged high vacuum used in TEM is difficult.

In this Letter, we introduce the fabrication of liquid cells for TEM using multilayer graphene sheets as the window material. The multilayer graphene windows have enhanced in-plane elasticity and excellent durability, while the defect-induced leakage of liquid is minimized; this allows us to encapsulate and protect hydrated samples that have uneven contour lines in a liquid with a thickness that is comparable to the size of the biological structure. As a result, we perform in situ TEM with high contrast due to significantly reduced background scattering. We use the graphene liquid cell (GLC) to directly observe structures of $\mathrm{H} 3 \mathrm{~N} 2$ influenza viruses in a buffer solution using TEM. We show that bright field TEM imaging at room temperature reveals structural details of viruses at nanometer scale spatial resolution, using low electron doses that are comparable to those used in conventional cryo-TEM. We also use a GLC to encapsulate whole cells and study the same samples with both TEM and fluorescence light microscopy while maintaining the cells in their native wet condition. We expect that our approach demonstrated in this paper can provide technical insights to researchers interested in in situ high-resolution TEM imaging of many different types of delicate specimens in their native liquid environments.

We prepare GLCs for different types of samples by encapsulating liquid phase specimens between two laminated sheets of multilayer graphene as schematically shown in Figure 1. A multilayer graphene sheet with a desired number of stacks from 3 to 10 is synthesized on a copper foil by chemical vapor deposition (CVD). The selection of the thickness of the multilayer graphene sheet depends on the dimensions of the specimen. Thinner graphene sheets have low out-of-plane stiffness and can encapsulate an object more conformally. This leads to a reduction of extra liquid between the windows and
GLC of viruses

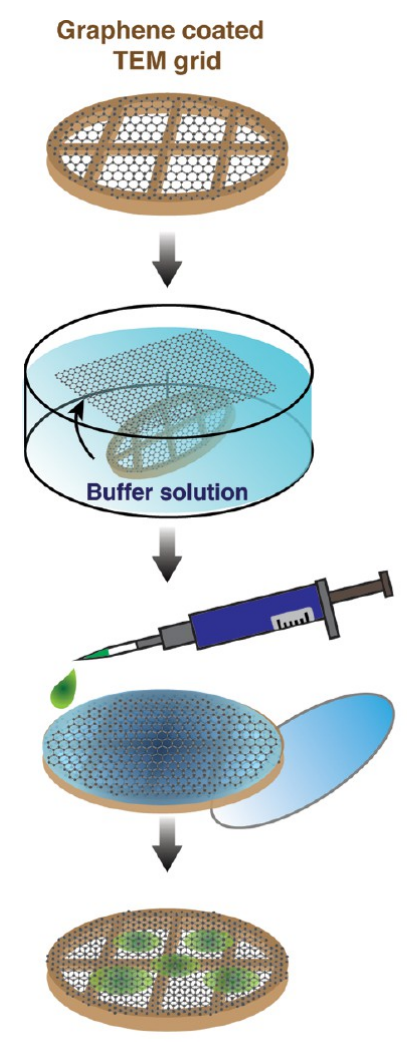

Cells cultured on graphene coated TEM grid
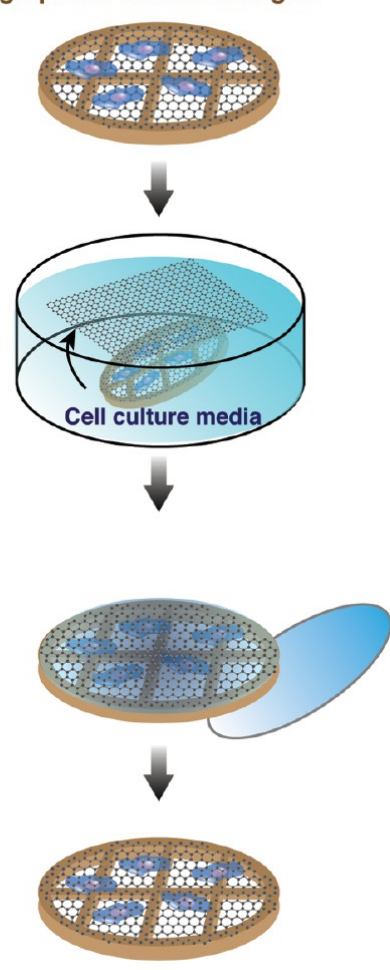

GLC of cells
Figure 1. GLC sample preparation for TEM observation. For GLC preparation of virus samples, free-standing multilayer graphene is lifted out from the surface of the buffer solution by graphene transferred TEM girds. A small amount of the sample solution is dropped on the side of the graphene cover along with the blotting the extra buffer solution by applying a filter paper on the opposite side of the TEM grid. Due to the surface tension of the graphene surface and a capillary force, the particle solution infiltrates and mixes with the buffer solution in between two multilayer graphene sheets. Procedures for GLC of cultured cells are accomplished by lifting out the free-standing multilayer graphene floated on the surface of the culture media solution by graphene transferred TEM girds with cells.

reduces unwanted scattering of the electron beam. Coverings made from more graphene sheets reduce leaking that can occur through defects and dislocations, but have increased stiffness. To fabricate a GLC, the direct transfer method is used to transfer a multilayer graphene sheet onto a TEM grid coated with a holey amorphous carbon film. ${ }^{27}$ The size of the holes on the supporting film limits the area where the graphene windows are fully unsupported. Next, a multilayer graphene sheet is grown on a copper foil by CVD, and left free-floating by direct wet chemical etching of a copper foil substrate as shown in Figure S1. This free-floating sheet is then transferred onto the surface of the aqueous solution to be encapsulated using an edge of a glass slide. The solution sample is encapsulated by lifting out the free-standing multilayer graphene with the previously prepared graphene-transferred TEM grid. To encapsulate a solution sample with a limited volume, such as microliter $\mathrm{H} 3 \mathrm{~N} 2$ virus solution, an appropriate buffer solution is first encapsulated between two multilayer graphene sheets. Then, we add a small volume of the virus solution on the edge of the TEM grid. The solution diffuses into the buffer solution between the two graphene sheets. Simultaneous blotting on the opposite side by a filter paper pulls the added solution between 
the graphene sheets and promotes the attachment of the two graphene sheets resulting in liquid pockets encased in multiple positions on the TEM grid. For imaging adherent epithelial cells, cells are first cultured on graphene coated TEM grids. This TEM grid is used to lift off the free-standing multilayer graphene sheet from the surface of the culture media. For encapsulation of nonadherent cells such as Bacillus subtilis, a free-standing multilayer graphene sheet can be also transferred onto the surface of cell suspension followed by directly lifting out the free-standing graphene sheet by multilayer graphene coated TEM grids. For encapsulation of both adherent and suspension cell, blotting by a filter paper can be also accompanied if the excess amount of solution prohibits successful adhesion between two graphene sheets.

The image resolution achievable in liquid phase TEM is directly proportional to the thickness of the liquid layer and windows. Image resolution deteriorates as samples and windows become thicker. ${ }^{28}$ Since two graphene sheets of GLCs presumably follow the contours of the top and bottom morphologies of encapsulated wet samples, the thickness of the entire space including samples, liquid, and windows would be of the same order of magnitude as the thickness of the sample itself. As a result, GLC can minimize loss of image resolution while simultaneously reducing the background in TEM images. Note that the entire thickness of the liquid layer in conventional $\mathrm{Si}_{3} \mathrm{~N}_{4}$ liquid cells is predetermined by the thickness of a spacer between two windows, and as a result, the extra aqueous solution above the embedded samples lowers the resolution.

It is also facile to fabricate reliable liquid cells with multilayer graphene sheets due to their mechanical durability and resulting ease of handling on the surface of the solution. The multilayer structure reduces the risk of sample rupture during handling and measurement, and the relatively small amount of encapsulated solution does not pose a threat to the TEM equipment. We maintain wet conditions in a high vacuum during TEM observation, typically $10^{-4} \mathrm{~Pa}$ range, while performing iterative studies of wet samples with fluorescence light microscopy (FLM) and TEM regardless of the order of using the two microscopies. If increased mechanical stability of liquid cells is required, we replace one graphene multilayer window with a $\mathrm{Si}_{3} \mathrm{~N}_{4}$ film to make a $\mathrm{Si}_{3} \mathrm{~N}_{4}$ graphene liquid cell (SGLC, Figure S2).

As in cryo-TEM, TEM imaging of delicate biological structures is routinely conducted in low-dose mode, often supported by post-TEM image processing to improve the signal-to-noise ratio. The low dose minimizes denaturing of delicate samples by the imaging electron beam, while the image processing such as averaging enables sophisticated structures to be resolved. To illustrate the use of GLC for imaging biological samples, we investigate $\mathrm{H} 3 \mathrm{~N} 2$ influenza viruses in a buffer solution and study their structural details using low-dose TEM at the room temperature. The images captured by TEM operated with $120 \mathrm{kV}$ accelerating voltage reveal that individual viruses have heterogeneous shapes as presented in Figure 2a to $2 \mathrm{~d}$. In addition to spherical shapes (Figure 2b), peapod (Figure $2 \mathrm{c}$ ), and snowman shapes (Figure $2 \mathrm{~d}$ ) of $\mathrm{H} 3 \mathrm{~N} 2$ viruses are also observed. Common to all of those structures, viruses show a distinctive enveloped structure with a sub-10 nm membrane that can be resolved using TEM. In addition, the outer layer of the membrane has higher contrast, which is presumably due to the presence of membrane proteins such as hemagglutinin and neuraminidase. We often observe materials in the interior space
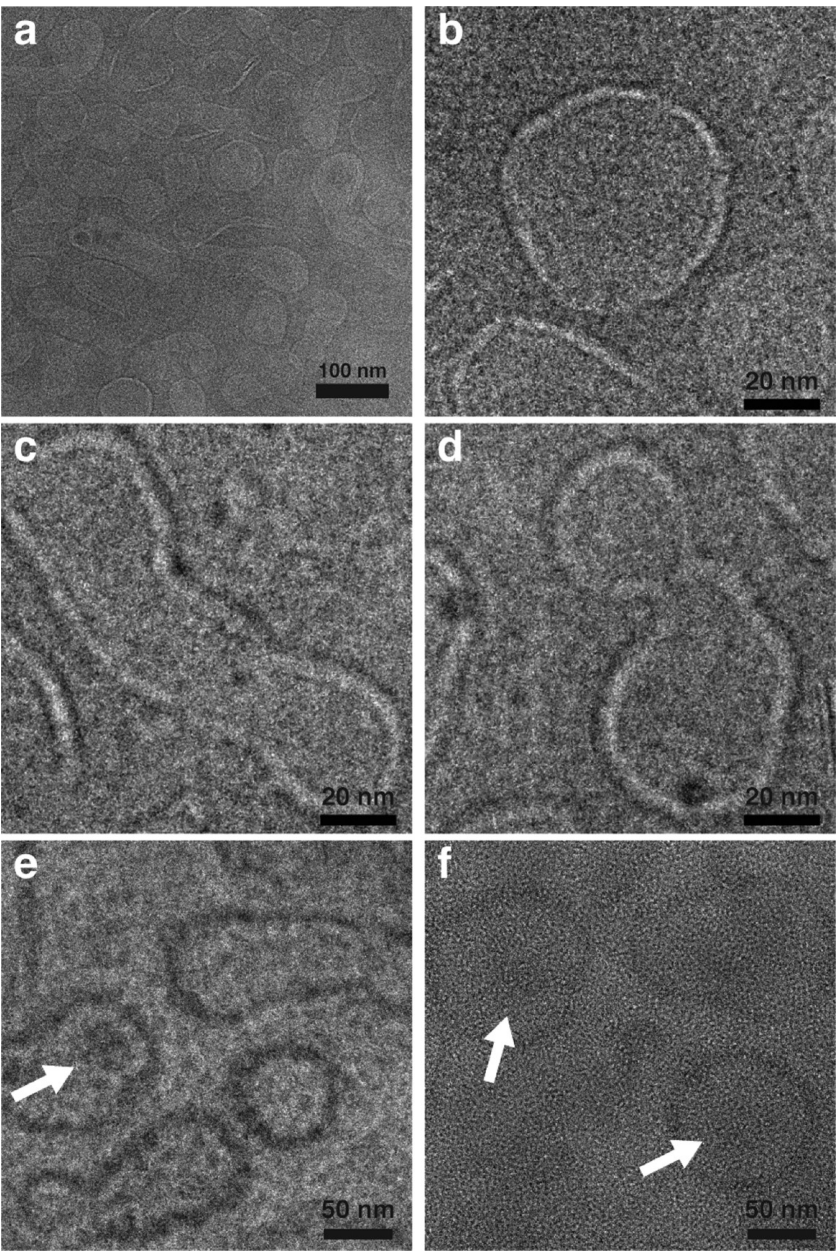

Figure 2. Low-dose TEM images of $\mathrm{H} 3 \mathrm{~N} 2$ viruses in GLCs that reveal structural details. (a) Low-resolution TEM image of $\mathrm{H} 3 \mathrm{~N} 2$ viruses in GLC. (b) Circular virus that shows a higher contrast for the outer membrane, which presumably indicates the presence of hemagglutinin and neuraminidase proteins. (c,d) Nonspherical viruses. The electron beam accelerated by $120 \mathrm{kV}$ and a camera with pixel resolution of $0.076 \mathrm{~nm}^{2} /$ pixel are used for (a) to $(d)$. (e,f) Viruses at very large under-focus and near focus in an aberration corrected TEM show the presence of the dense materials in the interior space, which are marked with arrows. The acceleration voltage was $80 \mathrm{kV}$ in an aberration corrected microscope and a camera with pixel resolution of $0.02 \mathrm{~nm}^{2}$ / pixel are used for (e) and (f).

that have a high contrast and become distinct in TEM images captured by TEAM I equipped with spherical and chromatic aberration correctors as shown with arrows in Figure 2e,f. An electron dose of approximately $5 \mathrm{e}^{-} / \AA^{2}$ is used to capture each TEM image; this is at the lower bound of the electron dose used in typical cryo-TEM. The contrast resolution of viruses is sufficient to distinguish the double-layer structure of the membrane at a defocus level of $3 \mu \mathrm{m}$ under-focus. Using graphene windows significantly enhances contrast resolution; viruses between graphene windows show distinct contrast, while ones on the amorphous carbon film are blurred and structures cannot be resolved (Figure S3). These results highlight the benefits of using a GLC.

Improved image contrast is achieved using higher electron dose, but this can lead to sample degradation; these competing priorities must be balanced to successfully image living samples. We demonstrate that TEM imaging in GLCs preserves contrast 
resolution and that the shape of viruses during sequential image captures with $5.48 \mathrm{e}^{-} / \AA^{2}$ per each image. Six sequential TEM images with the same electron dose and $1 \mathrm{~s}$ time interval are shown in Figure 3a. We also measure the intensity of individual pixels and show the distribution of pixel intensities for each corresponding image (Figure $3 \mathrm{~b}$ ). The average pixel intensity

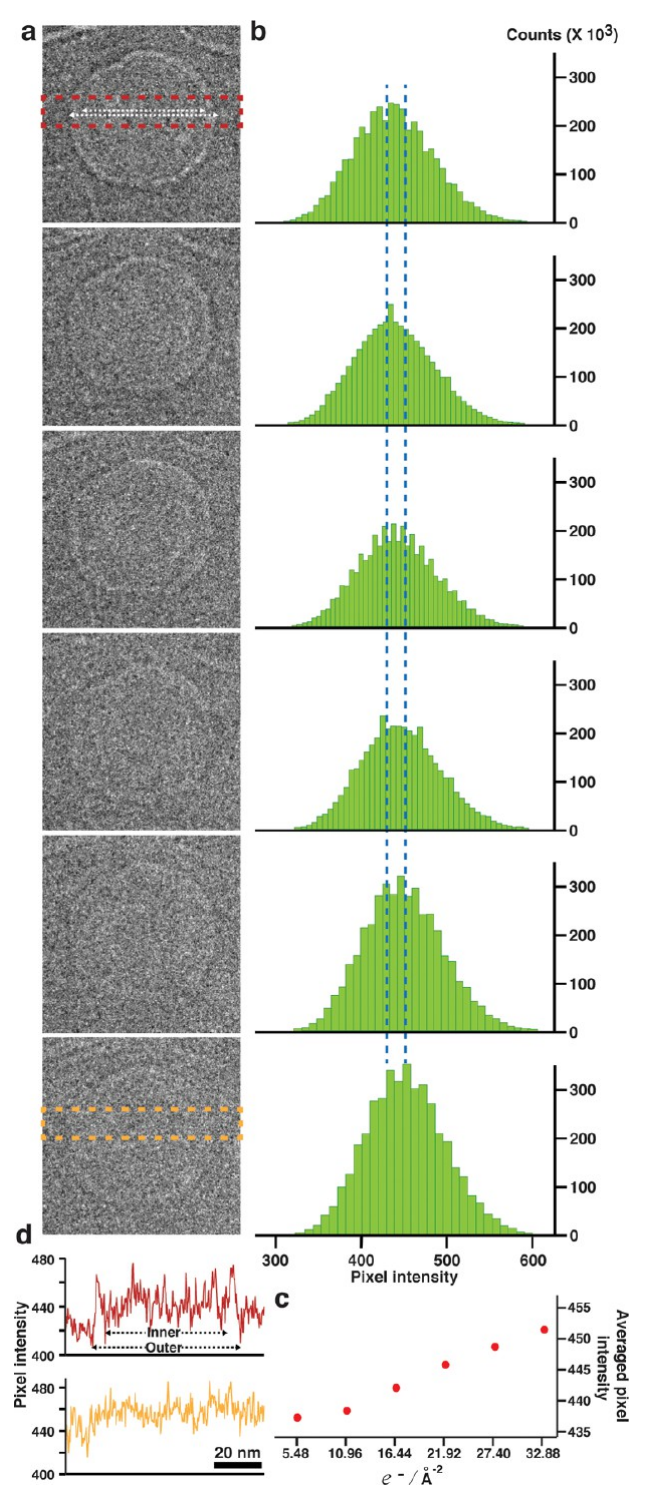

Figure 3. Contrast degradation of viruses in GLCs as a function of the total electron dose. (a) Sequential low-dose TEM snapshots that present the degradation of contrast resolution. Each image corresponds to the accumulation of $5.48 \mathrm{e}^{-} / \AA^{2}$. The electron beam accelerated by $120 \mathrm{kV}$ and a camera with pixel resolution of 0.076 $\mathrm{nm}^{2} /$ pixel are used. (b) Histograms of the total pixel intensity of each image in (a). Pixels in the images in (a) are displayed in 16-bit scale. (c) The average intensity value in the histogram in (b) is plotted as a function of the accumulated electron dose. Shifting to a greater value as increasing the accumulated electron dose means the image continuously loses dark contrast features. (d) Line profile integration in the red and orange boxes of the first and last images in (a). Line intensities are integrated along a vertical direction within the box. The line profile of the red box in the first image shows clear identification of the inner membrane, outer membrane, and membrane thickness of about $10 \mathrm{~nm}$. As the contrast resolution decreases in electron dose, the line profile loses such features. (vertical blue dashed lines in Figure 3b and a plot in Figure 3c) shifts from 437 to 452 as the accumulated electron dose increases. This indicates that features with darker contrast from denser material in the earlier images become less distinct as samples are exposed to higher electron dose. This observation can be directly seen in sequential TEM images, which continuously lose contrast resolution (Figure 3a). For example, we show the integration of line profiles (Figure 3d) within the boxes marked in the first and last images of Figure 3a. In the first image, the inner and outer boundaries of the membrane can be distinguished in the line profile integration plot, but they are less evident in the final image. Even with the reduction of contrast as a function of the total electron dose, we can still distinguish the bilayer structure of the viral membrane in the final image, which has a total accumulated electron dose of $32.88 \mathrm{e}^{-} / \AA^{2}$.

We also utilize the GLC to directly observe whole cells in liquid phase TEM at room temperature. Since the dimensions of cells are typically on the order of tens of micrometers, defectfree volume confinement by a window material must have a comparable dimension. Multilayer graphene sheets as windows can be successfully used for encapsulation of epithelial cells without leaking. We cultured Madin-Darby canine kidney, MDCK, cells on graphene coated TEM grids and successfully encapsulated them in GLCs without using further sample treatment such as fixation. Cells are maintained in a hydrated state in GLCs for TEM imaging. We first observe a typical GLC of MDCK cells stably transfected with a green fluorescence protein nuclear localization signal (GFP-NLS) construct, which results in a fluorescently labeled nucleus that can also be observed using FLM. Each square mesh of $70 \mu \mathrm{m}$ by $70 \mu \mathrm{m}$ contains multiple cells as is evidenced by the number of nuclei that can be counted in the overview image of GLC FLM in Figure 4a. Cell density can be controlled by the number of cells seeded onto the TEM grid and confirmed with the bright contrast from the nucleus in FLM. Additionally, the square mesh of the TEM grid, shown as dark contrast in Figure 4a, can be used as a reference for searching for a specific cell of interest for TEM imaging. TEM images with higher magnifications of the MDCK cell in GLC present zoomed in views of individual cells as shown in Figure $4 \mathrm{~b}$ to $4 \mathrm{~d}$. TEM images in higher magnifications in Figure $4 c, d$ show details from the subregions marked in Figure 4b. Overall, whole MDCK cells in GLCs exhibit high transparency in bright field imaging mode of TEM. The nucleus shows a much lower intensity due to its higher density, while the intracellular materials in the cell body are shown as light-gray patterns. We observe dense intracellular materials near the nuclei in Figure 4c probably because the cell contains a dense packing of various organelles close to the region near the nucleus. Intracellular material density decreases moving away from the nucleus such that the cell body becomes more transparent and thick filamentous structures become apparent in Figure 4d. We attribute this filamentous pattern to the cytoskeleton structure of the cell, which has densely polymerized filaments of monomer proteins. Near the cell boundary and near the neighboring cell on right side of Figure $4 \mathrm{~d}$, the filamentous structure becomes slightly more dense and entangled.

To confirm that GLCs sustain the wet condition of the whole cells for a prolonged time for TEM study, we observe the motion of $40 \mathrm{~nm} \mathrm{Au}$ nanoparticles, which are easily visible as dark dots due to their high density, located within MDCK cells in the GLC. MDCK cells are cultured on graphene coated 

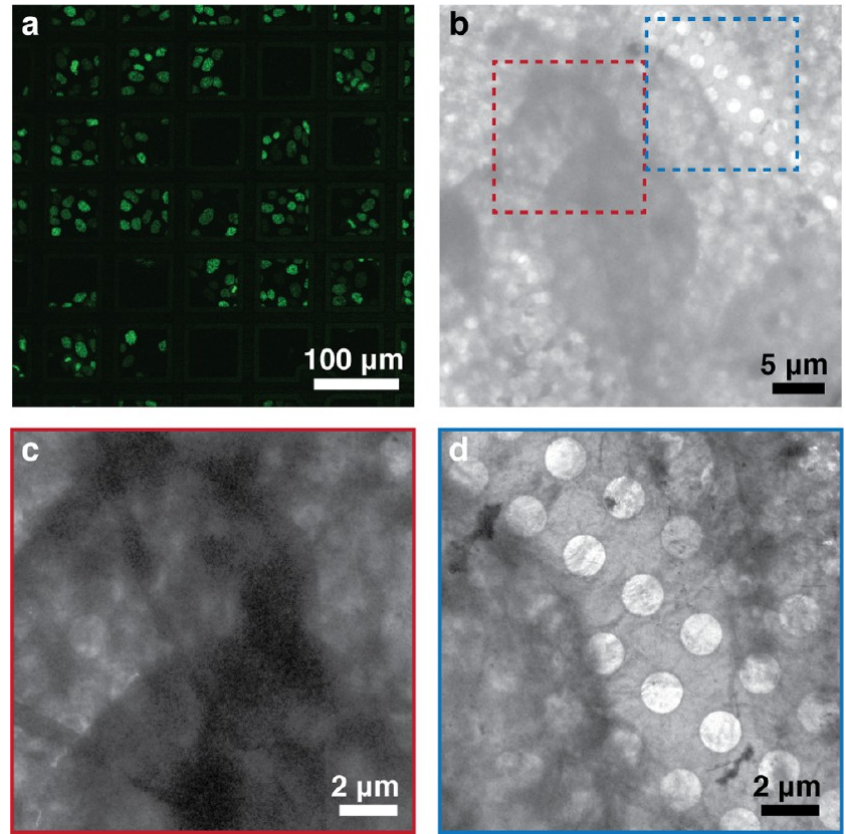

Figure 4. TEM observation of hydrated MDCK cell in the GLC. (a) Fluorescence image of live MDCK cells cultured on the graphene coated TEM grid. Nuclei of MDCK cells are stained with green fluorescence protein tagging and shown in green. The square mesh framework with the dark contrast indicates TEM grid mesh. The electron beam accelerated by $200 \mathrm{kV}$ and a camera with pixel resolution of $1311.23 \mathrm{~nm}^{2} /$ pixel are used. (b) Low magnification TEM image of MDCK cells in the GLC. (c,d) Magnified TEM images of subregions of the cell in (b). The nucleus shows high contrast, and the cellular material is shown as light-gray matter. The background with the bright circular pattern is from holey amorphous carbon film. The electron beam accelerated by $200 \mathrm{kV}$ and a camera with pixel resolution of $184.39 \mathrm{~nm}^{2} /$ pixel are used for (c) and (d).
TEM grids followed by incubation in the presence of $40 \mathrm{~nm} \mathrm{Au}$ nanoparticles pretreated in bovine serum albumin. Some $\mathrm{Au}$ nanoparticles are internalized within MDCK cells by nonspecific endocytosis; the remainders outside the cell body are removed by several washings with clean culture media before GLC encapsulation. The internalized Au nanoparticles can be seen in bright field TEM images of these cells, as indicated by red arrows in Figure 5a. Au nanoparticles are randomly distributed within the cellular matrix, and there is sufficient contrast to identify their positions while imaging through a graphene window. In a previous study of imaging $\mathrm{Au}$ nanoparticles in wet/fixed cells by using liquid phase scanning TEM (STEM) mode, the presence of $50 \mathrm{~nm}$ thick $\mathrm{Si}_{3} \mathrm{~N}_{4}$ windows required imaging a sample by STEM mode with a relatively high electron dose. ${ }^{19}$ Since the graphene window contributes less background, a lower electron dose in TEM mode can resolve individual $40 \mathrm{~nm}$ Au nanoparticles. If the $\mathrm{Au}$ particles are within a liquid environment, we expect the particle positions to fluctuate during TEM imaging. The measured step size distribution between frames of $0.1 \mathrm{~s}$ is shown in Figure $5 \mathrm{~b}$. Particles are presumably localized by nonspecific binding to intracellular organelles and thus exhibit a step motion of about $100 \mathrm{~nm}$, significantly less than that expected in Brownian motion or any sort of activated free motion. In addition to the fluctuations tracked on the $x$ and $y$ plane, particles also exhibit motion in other degrees of motion. The Au particle repeatedly appears either as a dark solid sphere or a dark halo with a bright core, as shown in Figure 5c; this indicates that the particle is either moving in and out of focus along a $z$-direction or rotating while at a constant defocus value throughout in situ observation. Our observation of Au particle dynamics not only confirms that the hydrated state of cells during TEM imaging is preserved but also suggests an opportunity to track intracellular events in situ. By exploiting the high contrast of Au particles, it is possible to label specific compartments of interest and track their real time
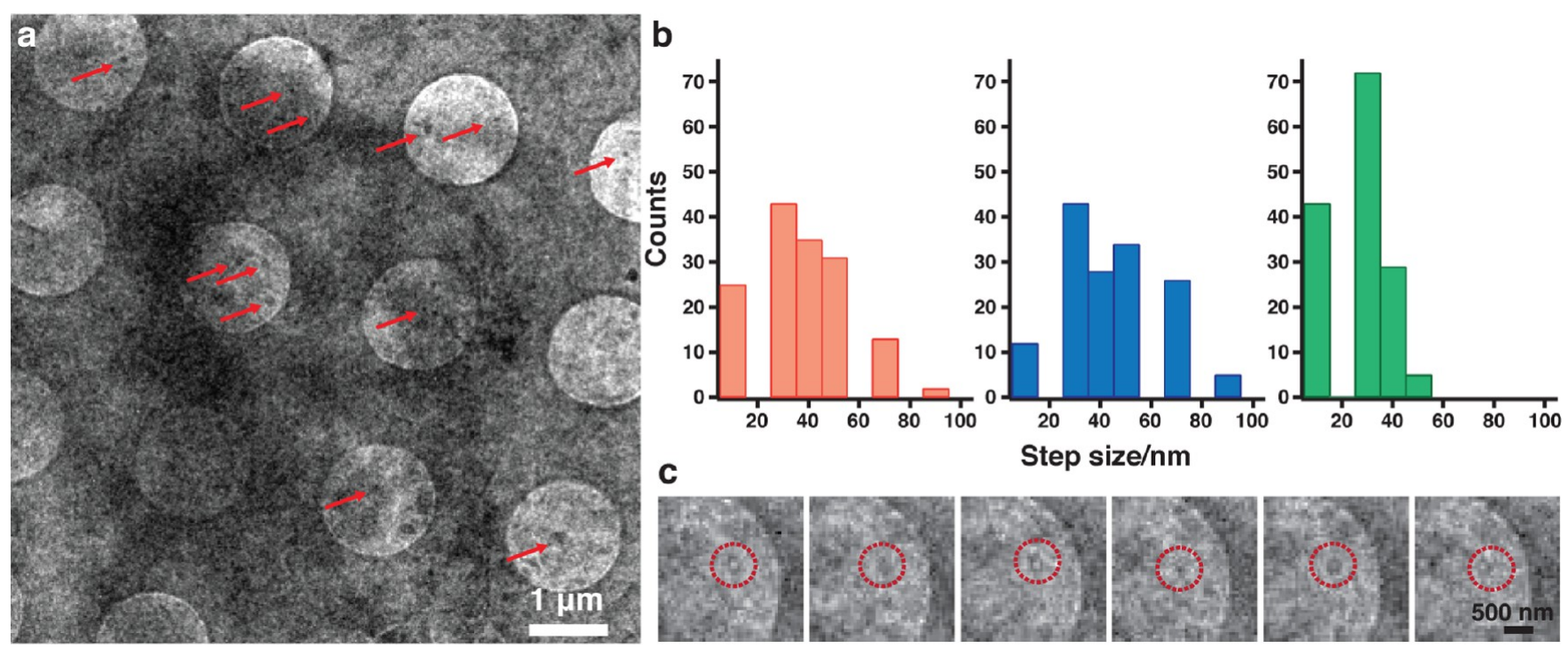

C
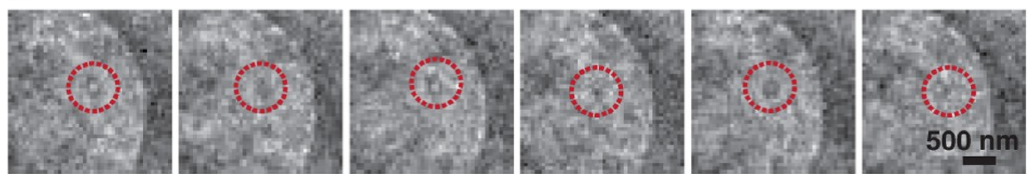

Figure 5. TEM observation of Au nanoparticles embedded in cellular matrix of the MDCK cell in the GLC. Cells are preincubated with $40 \mathrm{~nm}$ Au nanoparticles. (a) TEM image of the MDCK cell boundary where multiple Au nanoparticles digested by the cell are shown with red arrows. (b) Step-size counts of Au nanoparticles fluctuating in a cellular matrix. The $x$ and $y$ coordinates of Au particles are tracked from sequential images of a cell in the GLC, and 2D steps between frames are calculated. Time interval between frames is $0.1 \mathrm{~s}$. (c) TEM still snap-shots of an Au nanoparticle fluctuating with a nontranslational mode in a cellular matrix. Fluctuating Au nanoparticle in a red circle on each image repeatedly comes in and out of focus, which exhibits a solid dark circle and a dark halo with a bright center, respectively. The electron beam accelerated by $200 \mathrm{kV}$ and a camera with pixel resolution of $117.92 \mathrm{~nm}^{2} /$ pixel are used for (a) and (c). 

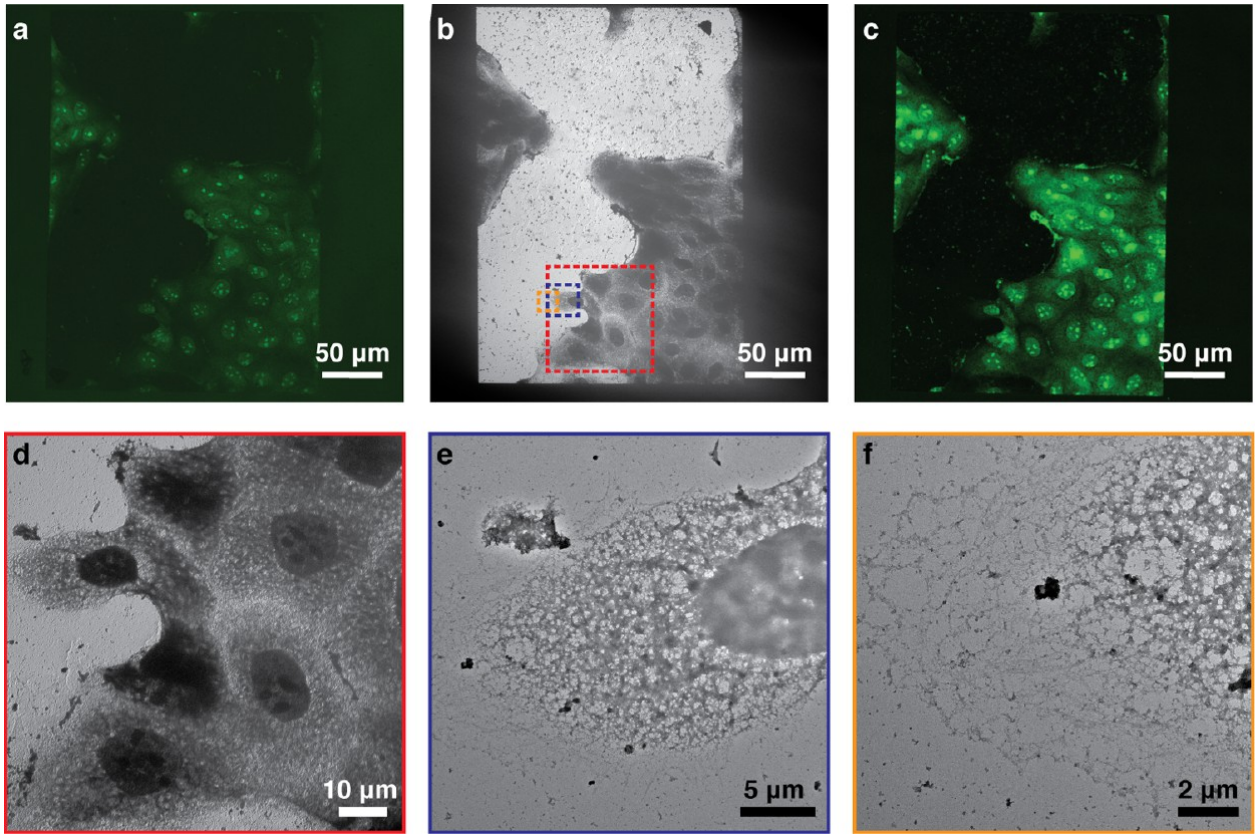

Figure 6. Iterative observation of MDCK cells by FLM and TEM. Cells for GLC are transfected to stain nuclei with green fluorescent proteins and cultured on a TEM grid fabricated with a $20 \mathrm{~nm}$ thick $\mathrm{Si}_{3} \mathrm{~N}_{4}$ window. Cellular membrane is removed by the treatment of detergent and fixed in a cytoskeleton stabilizing buffer. Graphene is placed on the top of cells prepared on a $\mathrm{Si}_{3} \mathrm{~N}_{4}$ TEM grid to keep the hydrated state of the specimen during iterative microscopic observation. (a) FLM image of groups of MDCK cells in the SGLC. (b) TEM image of MDCK cells in the same region shown in (a). (c) Post-TEM FLM image of MDCK cells in the same region shown in (a) and (b). (d-f) TEM images of MDCK cells with different magnifications. The colored box of each image corresponds to the area marked with the same color in (b). The electron beam accelerated by $200 \mathrm{kV}$ is used for (b) and (d-f) with the camera pixel resolution of $8521.51,405.68,42.46,10.24 \mathrm{~nm}^{2} /$ pixel, respectively.

position, taking advantage of the reduced sample degradation due to the low electron dose that can be used with the GLC. GLC is also used to study nonadherent cells in a buffer solution by TEM and details can be found in the Supporting Information.

The GLC provides an opportunity to exploit the highresolution capability of TEM to study low-mass biological samples in a liquid; however, gray scale TEM contrast is limited, making it difficult to study the spatial distributions and interactions of subcellular organelles. Such difficulties in conventional dry state TEM are traditionally overcome by combining TEM with FLM; however, this requires additional sample treatments that can in turn result in increased sample degradation. The capability of preserving a wet environment and the transparency of the graphene windows for both the electron beam and the light make GLC directly compatible with both FLM and TEM. We demonstrate this potential by studying groups of MDCK cells in GLC by FLM and cytoskeleton structures of the same cells by TEM. Cells are cultured on a TEM grid with a $20 \mathrm{~nm}$ thick $\mathrm{Si}_{3} \mathrm{~N}_{4}$ film window, followed by staining for FLM (Figure S1). To investigate the details of low-density intracellular structures with improved contrast that are otherwise concealed within the background materials such as water, proteins, and the cellular matrix, we fix cells and remove the cellular membrane by detergent treatment before encapsulation in SGLCs. We first observe a SGLC sample of MDCK cells using confocal FLM. A fluorescent image of MDCK cells shows that many cells are in contact and form groups (Figure 6a). After confocal microscopy, the SGLC is loaded in the TEM and imaged at low-resolution to identify the same region of interest. The low-resolution TEM image of the same region in Figure $6 \mathrm{~b}$ shows identical structure and morphology of the cells and their groups as does the FLM image in Figure 6a. This confirms that SGLC protects the sample integrity during sample transfer and the harsh imaging condition of TEM. We further confirm this by reimaging the same region using confocal FLM after TEM imaging. The confocal FLM image still shows the identical cell morphology without loss of fluorescence, as shown in Figure 6c.

Details of cytoskeleton structure are directly revealed in TEM images with high contrast and high spatial resolution. TEM images of local subcellular regions of a cell show structural details in local regions of the cell as shown in Figure $6 \mathrm{~d}-\mathrm{f}$ (TEM images that show exterior morphology of fixed cells in the GLC can be found in the Supporting Information). The color box for each image corresponds to the area marked with different colors in Figure 6b. The cytoskeleton of one cell forms a tight contact with neighboring cells, perhaps making intercellular adherens or tight junctions, which are hallmarks of epithelial cells and critical for collective cell motion ${ }^{29}$ (Figure 6d). The cytoskeleton along the boundary of each cell domain extends widely outward and forms networks of thick filament bundles as shown in Figure $6 \mathrm{~d}$. We also observe that cytoskeleton is a very dense, mesh-like network near the nucleus and extends outward with a descending degree of entanglement.

Our experimental results validate the feasibility of exploiting the high contrast and high resolution of TEM to image biological samples in a hydrated condition, without the harsh sample treatments that are typically required to use TEM to image low-mass materials. Nevertheless, confining the samples in the small volume of a GLC and the energy flux and radiolysis products produced by the electron beam irradiation can perturb the structural integrity of the sample. Technical improvements that continuously provide fresh media into a GLC can be further developed to achieve a less harsh liquid environment 
during a prolonged imaging period. It is unclear which mechanism causes the electron dose to disrupt the morphology of cells and viruses in a liquid environment, and this requires further investigation. Additionally, further investigation is required to determine whether TEM imaging in a liquid environment reveals different molecular and cellular structures than that in a dried, vacuum environment or under cryofreezing conditions. These structural differences are most likely to be evident in the 3D structure as the hydrated structure of the gel-like interior of the cell is likely to change substantially upon dehydration. Currently, only 2D TEM projection images have been acquired of GLC samples; overlap sample features along the projection direction limits the clear determination of internal structures. A combination of GLC with electron tomography will enable the realistic extraction of a 3D density map of internal cellular structures. ${ }^{9,30,31}$ Using multicolor labeling for different molecular complexes for correlative FLM and TEM will enable additional molecular specificity to be combined with the high spatial resolution of the TEM images of samples in GLC, further expanding its capabilities. Likewise, technical improvements should be accompanied by the careful optimization of the imaging conditions for different types of sample and required resolution.

GLC with multilayer graphene sheets provides multiple benefits for studying low-mass samples in their native hydrated condition. Reduced leaking, optimal elasticity, and mechanical rigidity of multilayer graphene sheets offer the capability to enclose wet samples with a wide range of length scales, from hundreds of nanometers for viruses to hundreds of micrometers for collections of epithelial cells. The hydration in GLC probably provides a more viable environment for encapsulated samples. A benign graphene surface can be readily used for mounting adherent and suspended cells. In addition, the high electrical/thermal conductivity of graphene and the presence of water effectively dissipate charges and energy produced by the incident electron beam. The dimension of the GLC is also compatible with conventional TEM holders, and the small amount of liquid encapsulated eliminates the possibility of damaging the TEM equipment.

In this Letter, we introduce the fabrication of GLC by using multilayer graphene sheets for TEM imaging of various wet biological samples. We demonstrated low-dose TEM imaging of GLCs of suspended viruses and, indeed, confirmed the achievement of TEM imaging with high contrast resolution in a buffer solution at room temperature. We also presented the use of GLC for study of wet whole cells by TEM in an intact culture environment and the iterative observation by FLM and TEM.

\section{ASSOCIATED CONTENT}

\section{S Supporting Information}

Methods for preparation of graphene liquid cells with multilayer graphene sheets and GLCs of biological samples and additional TEM images of H3N2 viruses, Bacillus subtilis, and fixed MDCK cells in GLCs. The Supporting Information is available free of charge on the ACS Publications website at DOI: 10.1021/acs.nanolett.5b01636.

\section{AUTHOR INFORMATION}

\section{Corresponding Author}

*E-mail: weitz@seas.harvard.edu.

\section{Notes}

The authors declare no competing financial interest.

\section{ACKNOWLEDGMENTS}

J.P., S.H.H., and D.A.W. acknowledge support from Amore Pacific. This work is also supported by the Harvard Materials Research Science and Engineering Center (DMR-1420570). The aberration-corrected electron microscopy was performed at the Molecular Foundry supported by the US Department of Energy DE-AC02-05CH11231. J.W.K. acknowledges support from the National Research Foundation of Korea (NRF) grant funded by the Korea government (MSIP) (No. 2008-0061891).

\section{REFERENCES}

(1) Smith, C. Nature 2012, 492, 293.

(2) Fernández-Suárez, M.; Ting, A. Y. Nat. Rev. Mol. Cell Biol. 2008, 9, 929.

(3) Claridge, S. A.; Schwartz, J. J.; Weiss, P. S. ACS Nano 2011, 5, 693.

(4) Schneckenburger, H. Curr. Opin. Biotechnol. 2005, 16, 13.

(5) Lichtman, J. W.; Conchello, J.-A. Nat. Methods 2005, 2, 910.

(6) De Cremer, G.; Sels, B. F.; De Vos, D. E.; Hofkens, J.; Roeffaers, M. B. J. Chem. Soc. Rev. 2010, 39, 4703.

(7) Jones, S. A.; Shim, S.-H.; He, J.; Zhuang, X. Nat. Methods 2011, 8, 499 .

(8) Huang, B.; Babcock, H.; Zhuang, X. Cell 2010, 143, 1047.

(9) Saghi, Z.; Midgley, P. A. Annu. Rev. Mater. Res. 2012, 42, 59.

(10) MacLaren, I.; Ramasse, Q. M. Int. Mater. Rev. 2014, 59, 115.

(11) Leis, A.; Rockel, B.; Andrees, L.; Baumeister, W. Trends Biochem. Sci. 2009, 34, 60.

(12) Fridman, K.; Mader, A.; Zwerger, M.; Elia, N.; Medalia, O. Nat. Rev. Mol. Cell Biol. 2012, 13, 736.

(13) Dubochet, J. J. Microsc. 2012, 245, 221.

(14) Van Heel, M.; Gowen, B.; Matadeen, R.; Orlova, E. V.; Finn, R.; Pape, T.; Cohen, D.; Stark, H.; Schmidt, R.; Schatz, M.; Patwardhan, A. Q. Rev. Biophys. 2000, 33, 307.

(15) Zhou, Z. H. Curr. Opin. Struct. Biol. 2008, 18, 218.

(16) Li, X.; Mooney, P.; Zheng, S.; Booth, C. R.; Braunfeld, M. B.; Gubbens, S.; Agard, D. A.; Cheng, Y. Nat. Methods 2013, 10, 584.

(17) Batson, P. E.; Dellby, N.; Kribanek, O. L. Nature 2002, 418, 617.

(18) Gilmore, B. L.; Showalter, S. P.; Dukes, M. J.; Tanner, J. R.; Demmert, A. C.; McDonald, S. M.; Kelly, D. F. Lab Chip 2013, 13, 216.

(19) De Jonge, N.; Peckys, D. B.; Kremers, G. J.; Piston, D. W. Proc. Natl. Acad. Sci. U.S.A. 2009, 106, 2159.

(20) Proetto, M. T.; Rush, A. M.; Chien, M.-P.; Abellan Baeza, P.; Patterson, J. P.; Thompson, M. P.; Olson, N. H.; Moore, C. E.; Rheingold, A. L.; Andolina, C.; Millstone, J.; Howell, S. B.; Browning, N. D.; Evans, J. E.; Gianneschi, N. C. J. Am. Chem. Soc. 2014, 136, 1162.

(21) Mirsaidov, U. M.; Zheng, H.; Casana, Y.; Matsudaira, P. Biophys. J. 2012, 102, L15.

(22) Mirsaidov, U. M.; Zheng, H.; Bhattacharya, D.; Casana, Y.; Matsudaira, P. Proc. Natl. Acad. Sci. U.S.A. 2012, 109, 7187.

(23) Holtz, M. E.; Yu, Y.; Gao, J.; Abruña, H. D.; Muller, D. A Microsc. Microanal. 2013, 19, 1027.

(24) Chen, Q.; Smith, J. M.; Park, J.; Kim, K.; Ho, D.; Rasool, H. I.; Zettl, A.; Alivisatos, A. P. Nano Lett. 2013, 13, 4556.

(25) Yuk, J. M.; Park, J.; Ercius, P.; Kim, K.; Hellebusch, D. J.; Crommie, M. F.; Lee, J. Y.; Zettl, A.; Alivisatos, A. P. Science 2012, 336, 61.

(26) Wang, C.; Qiao, Q.; Shokuhfar, T.; Klie, R. F. Adv. Mater. 2014, 26, 3410 .

(27) Regan, W.; Alem, N.; Alemán, B.; Geng, B.; Girit, C.; Maserati, L.; Wang, F.; Crommie, M.; Zettl, a. Appl. Phys. Lett. 2010, 96, 113102.

(28) De Jonge, N.; Ross, F. M. Nat. Nanotechnol. 2011, 6, 695.

(29) Tambe, D.; Hardin, C.; Angelini, T. Nat. Mater. 2011, 10, 469. 
(30) De Jonge, N.; Sougrat, R. Microsc. Microanal. 2010, 16, 54.

(31) Trasobares, S.; Lõpez-Haro, M.; Kociak, M.; March, K.; De La Peña, F.; Perez-Omil, J. A.; Calvino, J. J.; Lugg, N. R.; D’Alfonso, A. J.; Allen, L. J.; Colliex, C. Angew. Chem., Int. Ed. 2011, 50, 868. 